\title{
Promoting long-term weight control: does dieting consistency matter?
}

\author{
AA Gorin ${ }^{1,2}$, S Phelan ${ }^{1}$, RR Wing ${ }^{1}$ and JO Hill ${ }^{3}$ \\ ${ }^{1}$ Weight Control and Diabetes Research Center, Brown Medical School/The Miriam Hospital, USA; ${ }^{2}$ University of \\ Massachusetts Dartmouth, USA; and ${ }^{3}$ University of Colorado Health Sciences Center, USA
}

OBJECTIVE: The present study examined whether long-term weight loss maintenance is enhanced by maintaining the same diet regimen across the week and year or by dieting more strictly on weekdays and nonholiday periods than at other times.

METHOD: National Weight Control Registry participants $(N=1429)$ indicated on an eight-point scale whether they dieted more strictly on weekends than weekdays, adhered to the same diet regimen throughout the week, or dieted more strictly on weekdays. Participants responded to a similar question about holiday and vacation eating. Participants were then followed prospectively to determine whether scores on these questions were related to self-reported weight regain over the subsequent 12 months.

RESULTS: There was a linear relationship between scores on the dieting consistency questions and weight change over the 1-y period ( $P^{\prime} s<0.01$ ), with smaller weight gains in those who reported more consistency. Participants who reported a consistent diet across the week were 1.5 times more likely to maintain their weight within 5 pounds over the subsequent year $(O R=1.58$, 95\% Cl: 1.2-2.2) than participants who dieted more strictly on weekdays. A similar relationship emerged between dieting consistency across the year and subsequent weight regain.

CONCLUSION: Dieting consistency appears to be a behavioral strategy that predicts subsequent long-term weight loss maintenance.

Keywords: dieting consistency; weight maintenance

\section{Introduction}

Short-term weight loss can be produced through a variety of methods; however, long-term weight maintenance is rarely achieved. In clinic-based programs, only $60-70 \%$ of weight lost during treatment is maintained at 1-y post-treatment and nearly all weight is regained within $3 \mathrm{y}^{1,2} \mathrm{~A}$ handful of randomized-controlled trials have explored promising strategies for improving maintenance, such as extending the length of treatment, ${ }^{3}$ emphasizing problem-solving skills, ${ }^{4}$ increasing the amount of exercise,, 5 and providing financial incentives for weight maintenance. ${ }^{6,7}$ While these modifications have resulted in some improvement, long-term weight control remains a significant challenge.

An alternative approach to understanding weight maintenance is to study the habits and behaviors of individuals who have been successful at long-term weight control. The
National Weight Control Registry (NWCR) is a database of over 3000 individuals who have lost at least 30 pounds and kept the weight off for at least $1 \mathrm{y} .{ }^{8}$ Registry participants are followed prospectively at yearly intervals and provide detailed information about their weight, eating habits, and exercise patterns. Four common characteristics about successful weight maintenance have emerged from this unique sample: NWCR participants eat a low-calorie, low-fat diet, achieve a high level of physical activity, weigh themselves frequently, and eat regular meals, including breakfast. $^{8-10}$

The present study utilized the NWCR to examine whether maintaining the same diet regimen across the week and year promotes weight control or if dieting more strictly on weekdays and/or nonholidays is more conducive to longterm maintenance. Conceptually, it is not clear which pattern to recommend to patients. Allowing some flexibility on weekends, holidays, and vacations might reduce boredom, a major precipitant to dieting lapses, ${ }^{11,12}$ and be more realistic from a long-term perspective. However, flexibility might also increase exposure to high-risk situations, creating more opportunity for loss of control. 
The present study used the NWCR to determine whether the amount of consistency in diet regimens across the week and year is related to subsequent weight control. At year 1, Registry participants were asked whether they maintained a consistent diet regimen across the week or were stricter on weekdays or weekends. A similar question was asked about holiday and vacation eating $v s$ eating during the rest of the year. The answers to these questions were used to predict weight change over the following year prospectively.

\section{Materials and methods Participants}

To participate in the NWCR, individuals must be at least $18 \mathrm{y}$ old, and have maintained at least a 30-pound weight loss for at least $1 \mathrm{y}$. Volunteers for the Registry are recruited on an ongoing basis through advertisements on national and local television, radio, and magazine and newspapers. Participants are not compensated for their involvement in this longitudinal study.

The main variable of interest, consistency in diet regimen, was assessed $1 \mathrm{y}$ after entry into the Registry (these questions were not included in the assessment packet that participants fill out when they first enter the registry). Thus, for this study, we limited the sample to NWCR participants who at year 1 still met the entry criteria for the Registry (had lost $\geq 30$ pounds), were weight stable (defined as gaining 5 pounds or less over the past year), had completed the consistency questions, and denied pregnancy on self-report. Since we were interested in predicting weight change from years 1 to 2, we further limited the sample to NWCR participants who had reached their 2-y assessment point. Of the 1538 participants meeting the year 1 criteria and eligible for the year 2 assessment, 1429 had year 2 weights. Independent $t$-tests revealed that participants with year 2 weights were significantly older, had lower BMIs, and had maintained their weight for a longer period than participants who withdrew prior to the year 2 assessment (all $P$ 's $<0.05)$. There were no differences between participants with and without year 2 weights on the dieting consistency questions $\left(P^{\prime} \mathrm{s}>0.20\right)$.

\footnotetext{
Assessments

Demographic and weight history. Participants provided basic demographic information at entry into the Registry. Self-reported weight in pounds was also collected at entry into the Registry and at years 1 and 2 . The reliability and validity of self-reported weight in Registry participants has been reported previously. ${ }^{9}$ The primary dependent variable, weight change, was calculated by subtracting weight at year 1 from weight at year 2. Participants who reported a weight loss or gain over the 1-y period that was greater or less than 3 s.d.'s above the mean (gain or loss $\geq 12.01 \mathrm{~kg}$ ) were excluded from analyses because of concerns about their validity $(n=27)$.
}

Dieting consistency. Dieting consistency was assessed at year 1 with two questions, 'Do you maintain the same diet regimen on weekends as you do on weekdays?' (eight-point Likert scale, where $1=$ much stricter on weekends, $4=$ same on weekends and weekdays, and $8=$ much stricter on weekdays) and 'Do you maintain the same diet regimen on holidays and vacations as you do during the rest of the year?' (eight-point Likert scale, where $1=$ much stricter on holidays and vacations, $4=$ same on holidays and vacations as rest of the year, and $8=$ much stricter rest of the year). These questions have adequate test-retest reliability over a 2-y period in NWCR participants (0.67. weekend question; 0.68 holiday question).

Statistics. SPSS 11.0 for Windows was used for all analyses. An analysis of variance was used to examine changes in weight from years 1 to 2 . Post hoc contrasts were conducted and covariates entered into the models, when appropriate.

\section{Results}

On average, participants were $48.6 \pm 12.8$ y old, had a BMI of $24.1 \pm 3.8 \mathrm{~kg} / \mathrm{m}^{2}$, and had maintained at least a 30-pound weight loss for $7.9 \pm 9.3 \mathrm{y}$ (see Table 1 for further description). The distributions of participants' scores on the dieting consistency questions are displayed in Figure $1 \mathrm{a}$ and b. Few participants were stricter on weekends $(2.1 \%)$, and on holidays and vacations $(2.8 \%)$ than the rest of the week or year (score of 1-3). Owing to the small number of participants with scores of 1-3 (n's range from 2 to 19), these participants were excluded from further analysis. The dieting consistency scores of the remaining participants were distributed across the scale, with most participants reporting that their eating was the same (score of 4-5) on weekends and weekdays (59\%), and on holidays/vacations and the rest of the year (45\%). Participants reporting more consistency across both the week and year tended to be older $(P=0.000$ and 0.002 , respectively), and have maintained their weight loss for a longer duration at entry into the registry $(P=0.006$ and 0.001 , respectively). In addition, participants reporting more consistency across the year also reported larger initial

Table 1 Characteristics of participants at entry into the National Weight Control Registry

\begin{tabular}{llc}
\hline Characteristic & Mean \pm s.d. & $\%$ \\
\hline Gender (\% female) & & 76.1 \\
Age (y) & $48.58 \pm 12.84$ & \\
Ethnicity (\% white) & & 96.3 \\
Education (\% completed) & \\
$\quad$ High school & & 11.4 \\
$\quad$ Some college & & 26.6 \\
$\quad$ College & & 25.8 \\
$\quad$ Graduate or professional & 30.0 \\
BMl $\left(\mathrm{kg} / \mathrm{m}^{2}\right.$ ) & $24.06 \pm 3.80$ & \\
Duration $(\mathrm{y})$ at $13.6 \mathrm{~kg}$ weight loss criterion & $7.92 \pm 9.33$ & \\
\hline
\end{tabular}

$N 1429$. 


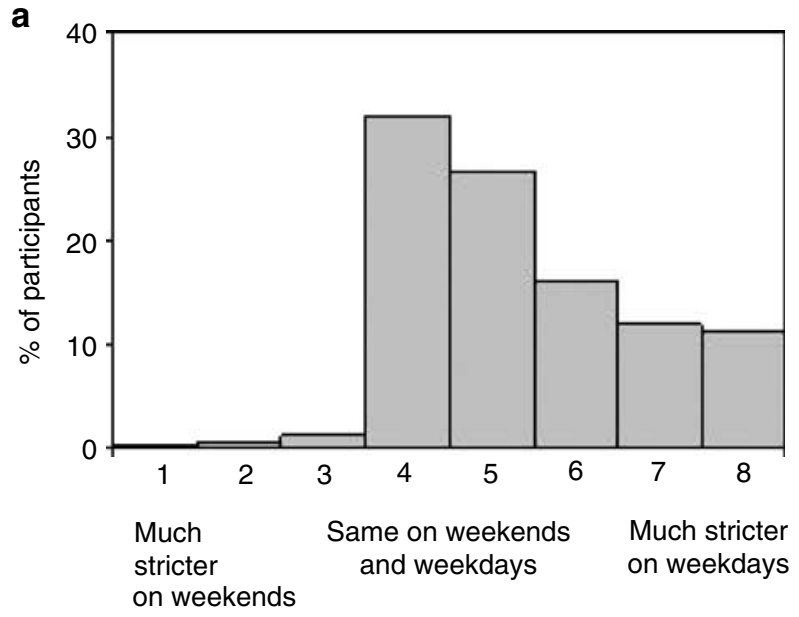

b

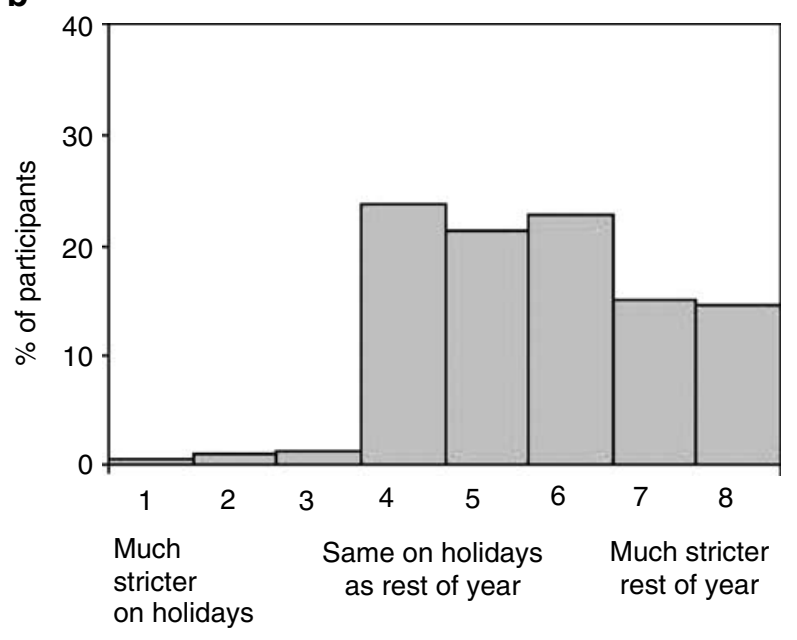

Figure 1 ( $a$ and b) Dieting consistency scores in NWCR participants.

weight losses $(P=0.000)$. Subsequent analyses controlled for these variables.

Scores on both the weekend and holiday consistency questions were related prospectively to weight change from years 1 to $2(P=0.01$ and 0.04 , respectively), with a strong linear trend in both analyses $(P=0.01$ and 0.006 , respectively, see Figure $2 \mathrm{a}$ and $\mathrm{b}$ ). Moreover, participants who maintained a consistent diet (scores of 4-5) were 1.5 times more likely to maintain their weight within 5 pounds over the subsequent year than participants who reported being stricter on weekdays (scores of 7-8) (OR $=1.58$, 95\% CI: $1.2-$ 2.2). A similar relationship was observed between dieting consistency across the year and the likelihood of regaining less than 5 pounds $(\mathrm{OR}=1.33,95 \% \mathrm{CI}$ : $1.0-1.8)$.

\section{Discussion}

The National Weight Control Registry is comprised of individuals who have lost a sizeable amount of weight and
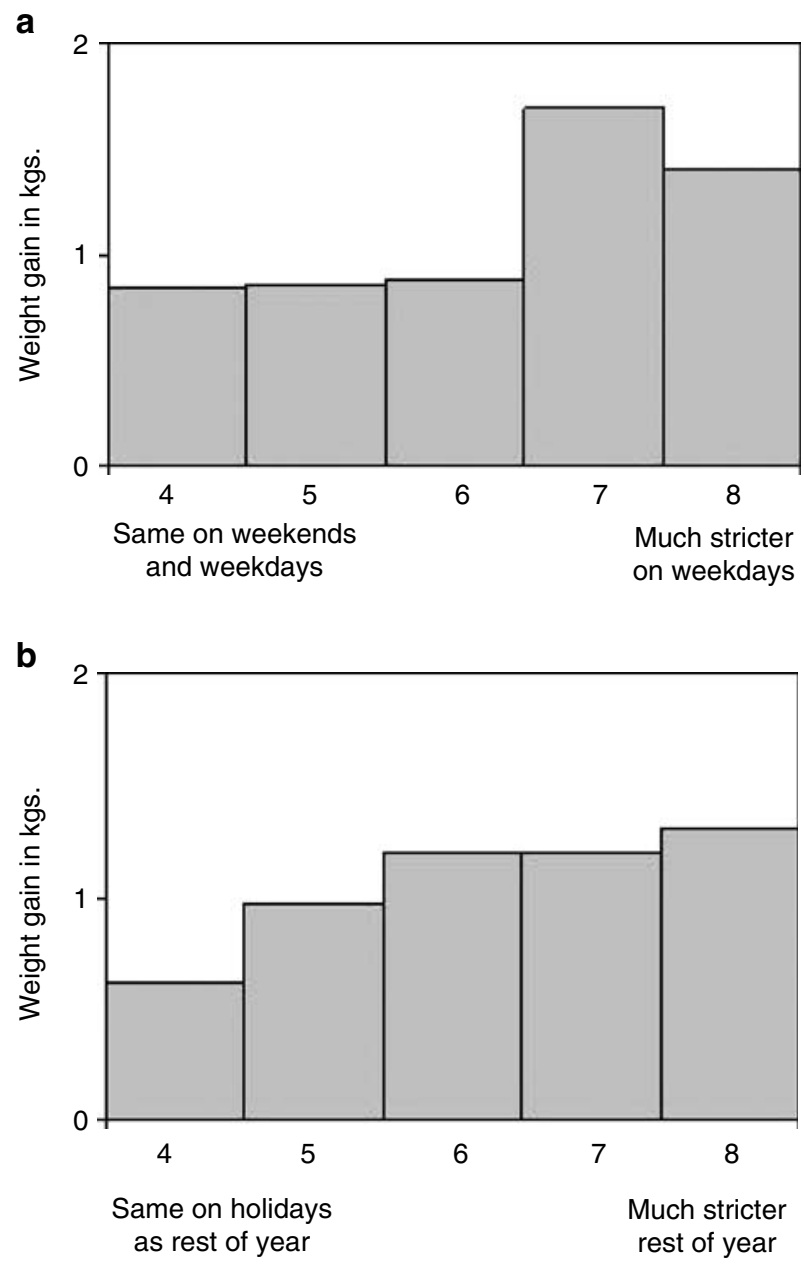

Figure 2 ( $a$ and $b$ ) Weight gain from years 1 to 2 for NWCR participants at each dieting consistency score.

kept it off for at least $1 \mathrm{y}$. Yet, even among these successful participants, regain over time is common. Few predictors of subsequent weight loss success have emerged in the NWCR. Two of the strongest predictors of long-term maintenance success are duration of weight maintenance and disinhibition; ${ }^{13}$ however, these are not behavioral strategies that can be taught to participants to assist in weight control. Behavioral variables such as caloric intake and amount of physical activity have not prospectively predicted weight control in the NWCR. ${ }^{13}$ The present study has identified a behavioral strategy, namely dieting consistency, that predicts weight regain prospectively. Participants who reported maintaining the same diet regimen across the week and year were more likely to maintain their weight loss over the following year than participants who dieted more strictly on weekdays and/or nonholiday periods. Given the correlational nature of this investigation, future studies are needed to determine whether training people to be more consistent across the week and/or year can improve their weight control success. 
Participants reporting the most consistency in their diet regimens at year 1 had maintained their weight loss for the longest time. One possible explanation for this finding is that consistency in diet regimen is a characteristic that develops naturally over time in maintainers. An alternative explanation is that individuals who were less consistent did not achieve long-term weight maintenance and thus were not represented in the NWCR. This latter explanation is supported by the finding that those who dieted more strictly on weekdays (or nonholidays) were most likely to gain weight when followed prospectively over the next year (and thus, over time, might no longer meet criteria for 'successful weight losers').

A major strength of this study was the large sample size afforded by the use of the National Weight Control Registry. By following individuals over time, the NWCR provides detailed information about the habits and characteristics of successful weight maintainers. A limitation of the study is the reliance on self-reported weights; however, self-reported weights are known to correlate highly with measured weights. ${ }^{9}$ In the present study, we evaluated the impact of dieting consistency on weight loss from years 1 to 2 of participation in the NWCR. This was necessary because the dieting consistency questions were not included in baseline assessments, and only in the year 1 assessment battery. Thus, the present study used participants who were weight stable from baseline to year 1 and still met Registry entry criteria at year 1 to evaluate the relationship between consistency at year 1 and weight change from years 1 to 2 .

It should be noted that weight regain in this sample over the year was rather small $(1.1 \pm 2.8 \mathrm{~kg})$. Further, all of the participants had maintained weight loss for substantial periods of time. The relationship between dieting consistency and weight regain could be different in participants who have maintained their weight loss for shorter periods of time and who may be prone to greater increases in weight gain.

The present study adds to the growing literature on weight maintenance. Previous examinations of NWCR participants have revealed that successful weight maintainers eat a lowcalorie, low-fat diet, achieve a high level of physical activity, weigh themselves frequently, and eat regular meals, including breakfast. ${ }^{8-10}$ This study suggests that individuals who have lost weight are more likely to maintain their weight loss over time if they maintain a consistent diet regimen across the week and year.

\section{Acknowledgements}

This research was supported by a grant from the Community Foundation for Southeastern Michigan (RR Wing).

\section{References}

1 Garner DM, Wooley SC. Confronting the failure of behavioral and dietary treatments for obesity. Clin Psychol Rev 1991; 11: 729780 .

2 Wing RR. Behavioral approaches to the treatment of obesity. In: Bray G, Bouchard C, James PT (Eds). Handbook of Obesity. Marcel Dekker: New York; 1997. 855-873.

3 Perri MG. The maintenance of treatment effects in the long-term management of obesity. Clin Psychol Sci Pract 1998; 5: 526-543.

4 Perri MG. Relapse prevention training and problem-solving therapy in the long-term management of obesity. J Consult Clin Psychol 2001; 69: 722-726.

5 Jeffery RW, Wing RR. The effects of an enhanced exercise program on long-term weight loss. Obes Res 2001; 9 (Suppl 3): 100S.

6 Jeffery RW, Wing RR, Thorson C, Burton LR, Raether C, Harvey J, Mullen M. Strengthening behavioral interventions for weight loss: a randomized trial of food provision and monetary incentives. J Consult Clin Psychol 1993; 61: 1038-1045.

7 Kramer FM, Jeffery RW, Snell MK, Forster JL. Maintenance of successful weight loss over 1 year: effects of financial contracts for weight maintenance or participation in skills training. Behav Ther 1986; 17: 295-301.

8 Wing RR, Hill JO. Successful weight loss maintenance. Annu Rev Nutr 2001; 21: 323-341.

9 McGuire MT, Wing RR, Klem ML, Hill JO. Behavioral strategies of individuals who have maintained long-term weight losses. Obes Res 1999; 7: 334-341.

10 Wyatt HR, Grunwald GK, Mosca CL, Klem M, Wing RR, Hill JO. Long-term weight loss and breakfast in subjects in the National Weight Control Registry. Obes Res 2002; 10: 78-82.

11 Nowalk MP, Wing RR, Koeske R. The effect of tasting food samples on the use of recipes distributed in nutrition counseling. J Am Diet Assoc 1986; 86: 1715-1716.

12 Smith C, Wing R, Burke L. Vegetarian and weight loss diets among young adults. Obes Res 2000; 8: 123-129.

13 McGuire MT, Wing RR, Klem ML, Lang W, Hill JO. What predicts weight regain in a group of successful weight losers? J Consult Clin Psychol 1999; 67: 177-185. 